\title{
Ocular toxoplasmosis: evaluation of lacrimal - specific secretory IgA levels in both patients with active and inactive phases of the disease
}

\author{
Luiz Felipe Lynch/ ${ }^{+}$, Maria Isabel Lynch, Rodrigo Santana do Nascimento Ferreira, \\ Mirelle Souza Leão Vasconcelos, Narjara Melo, Silvana Ferreira, Elizabeth Malagueño
}

Departamento de Oftalmologia, Laboratório de Imunopatologia Keizo Asami,

Universidade Federal de Pernambuco, Av. Prof. Moraes Rego 1235, 50670-901 Recife, PE, Brasil

\begin{abstract}
Ocular toxoplasmosis can result in recurrent uveitis. Studies have shown that a correlation between active ocular toxoplasmosis and the presence of anti-Toxoplasma gondii secretory IgA (SIgA) in tears. This study compares anti-T. gondii SIgA levels in patients' tears during the acute and inactive phases of toxoplasmic uveitis. Twenty-nine positive tear specific SIgA for T. gondii patients with acute toxoplasmic uveitis were selected and were followed-up for at least two years, when the anti-T. gondii SIgA tears levels were determined. Specific SIgA for T. gondii was negative in 22 patients (75.86\%) and positive in seven patients $(24.13 \%)$ of whom six (85.7\%) were followed over three years. Average SIgA levels during the acute phase are 1.54 and decrease significantly to $0.72(p=0.0001)$ during the inactive phase of disease. Because anti-T. gondii SIgA in the tear is negative in $75.86 \%$ of patients after the acute phase of infection, $\mathrm{T}$. gondii SIgA levels may be used as a complementary diagnostic marker for active ocular toxoplasmosis.
\end{abstract}

Key words: uveitis - ocular toxoplasmosis - secretory IgA - tears

Toxoplasmosis is one of the major causes of uveitis in the world and the primary cause of uveitis in Brazil (Silveira et al. 1988). Toxoplasmic uveitis is a problem for both clinicians and physicians because diverse clinical symptoms hinder diagnosis. In addition, uveitis is a recurrent disease in which approximately two-thirds of patients will be affected by new ocular lesions after periods of clinical improvement (Holland 2003). The diagnosis of toxoplasmic uveitis is based on clinical symptoms because no correlation between the levels of plasma antibodies and the patient's ocular symptoms has previously been established (Stanford et al. 2002). Intraocular fluid analysis may prove useful in diagnosis of selected patients. Garweg et al. (2004) concluded that immunoblotting for local, specific IgG and IgA antibodies can support clinical diagnosis of ocular toxoplasmosis in $70 \%$ of selected cases studied.

Secretory IgA (SIgA) reacting with Toxoplasma gondii in tears was described previously like the presence of anti $T$. gondii natural SIgA antibodies in patients without any sign of infection (Meek et al. 2000, 2002), also, the presence of anti-T. gondii specific SIgA in patients suffering from active ocular disease (Lynch et al. 2004). Lynch et al. (2009) have shown that it is possible to differentiate patients with active posterior uveitis caused by ocular toxoplasmosis from patients with uveitis resulting from other aetiologies by testing for anti-T. gondii $\mathrm{SIgA}$ in tears.

To correlate anti-T. gondii SIgA levels with the diagnosis of ocular toxoplasmosis, it is necessary to determine the levels of IgA at various stages of the dis-

+ Corresponding author:1flynch@hotlink.com.br

Received 6 February 2011

Accepted 14 July 2011 ease. It is known that anti-T. gondii IgA plasma levels rise during the acute phase of systemic infection and remain elevated for up to nine months decreasing afterwards to normal levels (Kodym et al. 2007). From an ophthalmologists' perspective, it is important to understand the behavior of SIgA during active ocular toxoplasmosis infection, as well as after the symptoms have cleared. Without understanding the behaviour of $\mathrm{SIgA}$, an accurate diagnosis will be difficult to obtain because many patients have recurrent disease. Therefore, prior abnormal results could impair interpretation of the diagnostic test. This study compares anti-T. gondii SIgA levels during the acute phase of ocular toxoplasmosis infection to the levels observed during the inactive phase of the disease.

Our patient cohort contained 29 patients with acute posterior uveitis caused by $T$. gondii. These patients were positive for T. gondii specific SIgA in their tears, as measured during the acute phase of the disease. Patients were followed for a period ranging from two-six years. During the inactive phase of ocular toxoplasmosis, a new sample of tear was taken and the SIgA levels were compared to those obtained during the acute phase. Patients with toxoplasmic uveitis were defined as carrying a gold standard lesion that is characterised by an active retinochoroidal lesion adjacent to a characteristic healed lesion (scars delimited with pigmented edges, with 1 or more foci) (Stanford et al. 2002, Garweg 2005).

Patients were 10 years of age or older, immunocompetent and had no other visible intraocular inflammatory disease of any aetiology after the initial event. This study was conducted at Clinics Hospital, Federal University of Pernambuco (UFPE) from February 2002-March 2006. To obtain a tear sample, a $5.0 \times 0.5 \mathrm{~cm} 4$-A Toyo filter paper was used. The paper was folded $0.5 \mathrm{~cm}$ from the end, inserted into the external third of the lower eyelid and then removed $5 \mathrm{~min}$ later. All samples were frozen 
at $-22^{\circ} \mathrm{C}$ after collection and until laboratory tests were performed. Tear tests were done at Keizo Asami Laboratory of Immunopathology at UFPE. Anti-T. gondii SIgA in the tears was analysed by enzyme linked immunosorbent assay using $T$. gondii $\mathrm{RH}$ crude antigens previously described by Lynch et al. (2004, 2009).

Student's statistical t-test and Pearson's chi-square test were used to compare paired samples. A p value of less than 0.05 was considered statistically significant. Stata software version 9.0 was used for analysis. The study was conducted under protocol 044/2004-CEP/ CCS of the UFPE Committee of Ethics.

The mean age of the study participants at the beginning of the study was 28.1 years (range $14-59$ years). The mean follow-up time was $3.5 \pm 1.2$ years, where $68.9 \%$ of the cases were re-evaluated at the second or third year after the acute phase and $31.1 \%$ at the forth or sixth year after. The number of patients with the disease in the right eye was $18(62.1 \%)$ and 11 patients (37.9\%) had the disease in the left eye (Table).

TABLE

Characterization of patients with ocular toxoplasmosis followed-up at Clinics Hospital, Federal University of Pernambuco

\begin{tabular}{lcc}
\hline Variables & $\mathrm{n}$ & $\%$ \\
\hline $\begin{array}{l}\text { Affected eye } \\
\text { Right }\end{array}$ & 18 & \\
$\quad$ Left & 11 & 62.1 \\
Follow-up time & & 37.9 \\
2 years & 03 & \\
3 years & 17 & 10.34 \\
4 years & 04 & 58.62 \\
5 years & 01 & 13.75 \\
6 years & 04 & 3.44 \\
\hline Total & 29 & 13.75 \\
\hline
\end{tabular}

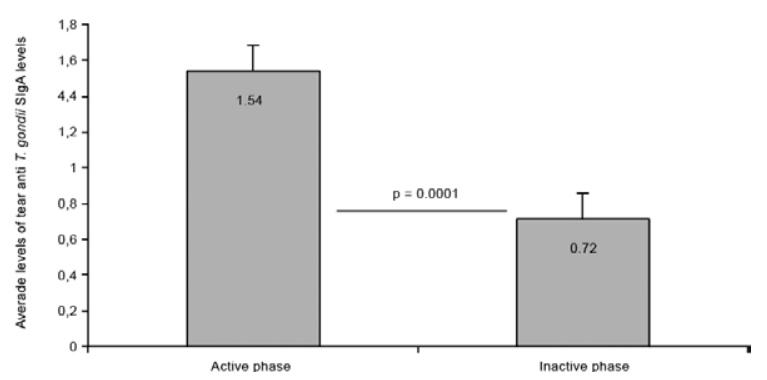

Fig. 1: average of tears anti-Toxoplasma gondii secretory $\operatorname{IgA}(\mathrm{SIgA})$ reactivity levels during both active and inactive phases of ocular toxoplasmosis in patients followed-up at Clinics Hospital, Federal University of Pernambuco.
Qualitative analysis of the data shows that out of the 29 patients positive for anti-T. gondii SIgA antibodies during the acute phase, 22 patients $(75.86 \%)[95 \%$ confidence interval (CI): 56.5-89.7] were negative and seven patients $(24.13 \%)$ (95\% CI: $10.3-43.5)$ were positive for anti-T. gondii SIgA during the inactive phase of the disease. Of the 22 negative patients, 14 (63.63\%) were re-evaluated between two-three years and eight $(36.36 \%)$ were re-evaluated between four-six years. Of the seven positive patients, six $(85.7 \%)$ had follow-up times from two-three years and just one (14.3\%) had a follow-up time of four years.

The average SIgA levels of all patients during their active and inactive phases were compared. Quantitative analysis showed a statistically significant reduction $(\mathrm{p}=$ 0.0001 ) in the average level of reactive SIgA from 1.54 in the acute phase (95\% CI: $1.26-1.82)$ to 0.72 in the inactive phase (95\% CI: 0.50-0.94) (Fig. 1).

It was compared the SIgA reactivity levels of the 22 negative patients tears samples in the active and in the inactive phase of infection. It was observed reduction of reactivity according to the time passed after the active phase. In Fig. 2, patient's SIgA levels were plotted against the time to follow-up. The sample size was too small to assess statistical significance.

The mean age of patients in the study with active phase of disease was 28.1 years. This shows that the patients studied tend to have recurrence of ocular toxoplasmosis between the second and fourth decades, as discussed by Holland (2004) and Lynch et al. (2008). A minimum follow-up time of two years was used to reduce the probability of detecting antibodies related to the acute event because serum IgM and IgA can be detected up to 18 months and nine months, respectively, following systemic toxoplasmosis (Kodym et al. 2007). Patients included in this study had at least one recurrence of acute toxoplasmosis, which was indicated by a scar associated with the active satellite lesion at the time of follow-up, and all were positive for tear SIgA specific for T. gondii during the acute phase of ocular toxoplasmosis.

In this study, to determine the reactivity of anti- $T$. gondii SIgA in the tears, we established a ratio between the absorbance obtained from patients' tears and the absorbance found in tears of normal individuals, whether

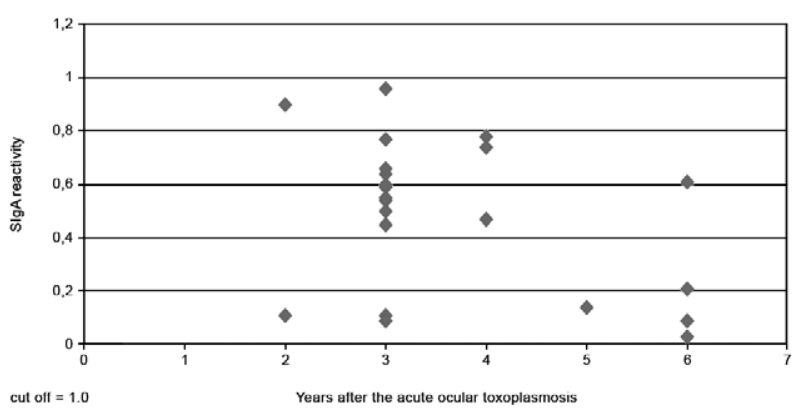

Fig. 2: anti-Toxoplasma gondii tears secretory $\operatorname{IgA}(\mathrm{SIgA})$ reactivity levels during the inactive phase of ocular toxoplasmosis in the 22 negative patients followed-up in different periods of time. 
of natural or residual origin. Antibody reactivity to $T$. gondii is described here as specific antibodies at concentrations above those found in individuals without toxoplasmosis. Reactivity of 1.0 or greater was considered specific SIgA reactivity.

Several mechanisms may explain a positive tear response in the case of reactivated toxoplasmic retinochoroiditis. The most probable explanation is the breakdown of ocular barriers that enable the exit of antigenpresenting cells from the eye. Local inflammation, both at the level of the retinal vessels and in the retinal pigment epithelium, could expose tachyzoite antigens to stimulate the clonal proliferation of memory B-lymphocytes. By circulating through the capillaries of the ocular mucosa, B-lymphocytes would be captured by high endothelial venules and would induce the release of anti-T. gondii specific SIgA into the tears. Another possibility, that complements the previous one, would be outflow of antigen-presenting cells from the eye globe, which would reach the submandibular lymph nodes, as shown by Egan et al. (1996) in mice, where specific Blymphocytes would be activated and might even reach the ocular mucosa, producing specific SIgA. Both mechanisms are shown in several diseases, according to the literature, but they have not been proven in cases of toxoplasmosis (Meek et al. 2003).

In this study, none of the patients, including the seven patients with persistent levels of anti-T. gondii $\operatorname{SIgA}$ in their tears, had signs of ocular inflammation, toxoplasmosis-related or from any other aetiology. The stimulus to maintain the production of specific SIgA for long periods can be either local or systemic. Considering that the ocular mucosa is not chronically colonized by $T$. gondii, the stimulus to maintain positive levels of specific SIgA has another origin. Here we consider two possibilities.

First, stimulus from cysts, ocular or from other tissues, could lead to a chronic presentation of Toxoplasma antigens through the asymptomatic breakdown of these cysts (Holland 2003). This would result in positive levels of SIgA being maintained in mucosa by circulating lymphocytes (Knop \& Knop 2007).

Another possibility is that chronic stimulation of the intestinal mucosa may occur due to continuous exposure to $T$. gondii, especially in locations with high prevalence of the parasite. The frequent uptake of tissue cysts or oocysts may stimulate memory B-lymphocytes in the intestinal mucosa to produce SIgA. When these activated lymphocytes reach the bloodstream through the lymphatic system, the lymphocytes can stimulate other mucosal surfaces to generate the production of specific antibodies (Gregory \& Filler 1987, Knop \& Knop 2007).

As seen in Fig. 2, anti-T. gondii SIgA levels tend to decrease with time. An increased study size with more patients followed for a longer period of time may confirm this trend.

To properly interpret the results of anti-T. gondii SIgA levels in the tears of patients with ocular recurrences, it is important to know the levels of SIgA during the acute phase of their disease. In the present study, there was a significant decrease in SIgA production between the acute and inactive phase of the disease in most of pa- tients. This demonstrates that lacrimal SIgA can be used as an acute phase marker since levels normalise after the uveitis event. For example, a female patient presented with active ocular toxoplasmosis and was positive for SIgA in June 2005. A tear sample was collected in April 2008 during an inactive period and she was found to be SIgA negative. In June 2008, she experienced recurrence of toxoplasmic uveitis and a new tear sample was taken. At this time, the patient was SIgA positive, indicating that the ocular surface received a new stimulus for the production of anti $T$. gondii SIgA during the reactivation of the ocular toxoplasmosis.

The results of this study show that anti-T. gondii specific SIgA is present during the acute phase of ocular toxoplasmosis but, in most patients, is absent during the inactive phase. This information will enable the use of anti-T. gondii specific SIgA levels as a diagnostic marker of acute toxoplasmic uveitis to facilitate a more accurate diagnosis of patients with uveitis.

\section{REFERENCES}

Egan RM, Yorkey C, Black R, Loh WK, Stevens JL, Woodward JG 1996. Peptide-specific T cell clonal expansion in vivo following immunization in the eye, an immune-privileged site. J Immunol 157: 2262-2271.

Garweg JG 2005. Determinants of immunodiagnostic success in human ocular toxoplasmosis. Parasite Immunol 27: 61-68.

Garweg JG, Garweg SD, Flueckiger F, Jacquier P, Boehnke M 2004. Aqueous humor and serum immunoblotting for immunoglobulin types $\mathrm{G}, \mathrm{A}, \mathrm{M}$, and $\mathrm{E}$ in cases of human ocular toxoplasmosis. J Clin Microbiol 42: 4593-4598.

Gregory RL, Filler SJ 1987. Protective secretory immunoglobulin A antibodies in humans following oral immunization with Streptococcus mutans. Infect Immun 55: 2409-2415.

Holland GN 2003. Ocular toxoplasmosis: a global reassessment. Part I: epidemiology and course of disease. Am J Ophthalmol 136: 973-988.

Holland GN 2004. Ocular toxoplasmosis: a global reassessment. Part II: disease manifestations and management. Am J Ophthalmol 137: 1-17.

Knop E, Knop N 2007. Anatomy and immunology of the ocular surface. In JY Niederkorn, HJ Kaplan (eds.), Immune response and the eye, Chemical immunology and allergy, Karger, Basel, p. 36-49.

Kodym P, Machala L, Rohácová H, Sirocká B, Malý M 2007. Evaluation of a commercial IgE ELISA in comparison with IgA and IgM ELISAs, IgG avidity assay and complement fixation for the diagnosis of acute toxoplasmosis. Clin Microbiol Infect 13: 40-47.

Lynch MI, Cordeiro F, Ferreira S, Ximenes R, Oréfice F, Malagueño E 2004. Lacrimal secretory IgA in active posterior uveites induced by Toxoplasma gondii. Mem Inst Oswaldo Cruz 99: 861-864.

Lynch MI, Malagueño E, Lynch LF, Ferreira S, Stheling R, Oréfice F 2009. Anti-Toxoplasma gondii secretory IgA in tears of patients with ocular toxoplasmosis: immunodiagnostic validation by ELISA. Mem Inst Oswaldo Cruz 104: 818-822.

Lynch MI, Moraes LFL, Malagueño E, Ferreira S, Cordeiro F, Oréfice F 2008. Características clínicas de 64 indivíduos portadores de uveítes posterior activa presumiblemente toxoplásmica en Pernambuco. Arq Bras Oftalmol 71: 43-48.

Meek B, Back JW, Klaren VN, Speijer D, Peek R 2002. Conserved regions of protein disulfide isomerase are targeted by natural $\operatorname{IgA}$ antibodies in humans. Int Immunol 14: 1291-1301. 
Meek B, Klaren VN, van Haeringen NJ, Kijlstra A, Peek R 2000. IgA antibodies to Toxoplasma gondii in human tears. Invest Ophthalmol Vis Sci 41: 2584-2590.

Meek B, Speijer D, de Jong PT, de Smet MD, Peek R 2003. The ocular humoral immune response in health and disease. Prog Retin Eye Res 22: 391-415.
Silveira C, Belfort R Jr, Burnier M Jr, Nussenblatt R 1988. Acquired toxoplasmic infection as the cause of toxoplasmic retinochoroiditis in families. Am J Ophthalmol 106: 362-364.

Stanford MR, Gras L, Wade A, Gilbert RE 2002. Reliability of expert interpretation of retinal photographs for the diagnosis of toxoplasmaretinochoroiditis. Br J Ophthalmol 86: 636-639. 